Results 11 women with FBSD (mean age 37 (31-47 years) underwent biliary sphincterotomy and intermittent Botox injections prospectively over a period of (27 (6-56) months), with a mean of 6 (2-11) Botox injections per patient, delivered at a mean of every 5 (2-11) months. A median Botox dose of 400 (100-600) units was used. Once symptoms became refractory to Botox injections, all patients underwent uncomplicated trans-duodenal surgical sphincteroplasty. On outpatient review over 12 months, 8 patients $(73 \%)$ reported complete relief of pain and were able to stop regular opioid analgesia and did not experience any further episodes of pain or hospital admissions. 3 patients reported partial relief of pain and needed to continue opioids. There were no further hospital admissions in this cohort either.

Conclusions Surgical sphincteroplasty is useful in managing refractory pancreaticobiliary pain in sphincterotomised patients with FBSD.

\section{PWE-56 EFFECT OF CHANGES IN GUT TRANSIT ON GI SYMPTOMS}

${ }^{1}$ Haider Khan*, ${ }^{2}$ mad Wazir, ${ }^{3}$ Malik Aamaz Khan, ${ }^{1}$ Stephen Lewis. ${ }^{1}$ Derriford Hospital Plymouth, Plymouth, UK; ${ }^{2}$ Royal Derby Hospital, Nottingham, UK; ${ }^{3}$ Princess Alexandra Hospital, Harlow, UK

\subsection{6/gutjnl-2021-BSG.318}

Introduction To evaluate the effect of induced changes in WGTT on symptoms.

Background Whole-gut transit-time (WGTT) has been shown to be faster in patients with anxiety and slower in those with depression. Abdominal symptoms are more common in patients reporting constipation and volunteers with induced constipation.

Methodology Healthy volunteers were randomly allocated to a baseline assessment period followed by another assessment period whilst taking either senna or loperamide. After a 2week washout volunteers were crossed over to receive the other intervention after a baseline assessment. Patients had their WGTT measured and were evaluated with symptoms Likart scales (range 0-100\%) for anxiety, bloating, hunger, quality of sleep, abdominal cramp and energy levels. Results were analysed parametrically with paired t-tests and Pearson's correlations.

Results Of the 18 healthy volunteers 12 were male. The mean age and BMI were 40 years and $25.6 \mathrm{~kg} / \mathrm{m}^{2}$ respectively. Mean baseline WGTT was $46 \mathrm{~h} \mathrm{SD} \pm 16 \mathrm{~h}$. Loperamide increased mean WGTT by $22 \mathrm{~h} \mathrm{SD} \pm 14 \mathrm{~h}, \mathrm{p}<0.001$ (95\% CI: 4-30), Senna reduced by $-14 \mathrm{~h} S \mathrm{SD} \pm 13 \mathrm{~h} \mathrm{p}=0.002$ (95\% CI: 6-22). Both loperamide (26\% (95\% CI 15-36) $\mathrm{p}<0.001)$ and senna $(12 \%(95 \%$ CI $0-24) \mathrm{p}=0.05)$ increased bloating. Loperamide increased abdominal cramps by $23 \%$ (95\% CI 11-35) $p=0.001$. No changes in the scores for the other parameters were noted. WGTT did not correlate with any symptom. Bloating correlated with cramps $(\mathrm{r}=0.85, \mathrm{p}<0.001)$, anxiety $(r=42, p=0.006)$, energy levels $(r=69, p<0.001)$ and quality of sleep $(\mathrm{r}=-0.55, \mathrm{p}<0.001)$.

Conclusions Increasing and decreasing WGTT resulted in bloating, though this did not directly correlate with WGTT. This suggests that it is the change in bowl habit that causes distress, not the direction of change. The presence of bloating correlated with increased anxiety, cramps, energy levels and poor sleep perhaps suggesting an interrelationship between these symptoms unrelated to WGTT.

\section{PWE-57 IS GUT-FOCUSED HYPNOTHERAPY AN EFFECTIVE TREATMENT OPTION FOR BRITISH SOUTH ASIANS WITH SEVERE REFRACTORY IBS?}

Syed S Hasan*, Peter J Whorwell, Dipesh Vasant. Manchester University NHS Foundation Trust, Manchester, UK

\subsection{6/gutjnl-2021-BSG.319}

Introduction Recent studies have confirmed a high worldwide prevalence of Irritable Bowel Syndrome (IBS) and suggest that cultural factors including; social taboos, stigmatisation, dietary factors, healthcare beliefs and communication gaps may contribute to disparities in the management of IBS between ethnic groups. It is unknown whether these factors contribute to outcomes of IBS treatments in different ethnic groups. In this context, we evaluated outcomes of gut-focused hypnotherapy in a South Asian population for the first time.

Methods Consecutive South Asian patients with refractory IBS that received 12 weekly sessions of gut-focused hypnotherapy (GFH) using the Manchester protocol were included. Patients were treated by a team including a therapist able to speak several South Asian languages. All patients prospectively completed the following questionnaires before and after GFH; IBS symptom severity score (IBS-SSS), Hospital Anxiety and Depression scale (HAD), Non-Colonic Symptom score and quality-of-life (QoL) score. The primary outcome measure was response to GFH defined by $\geq 50$-point reduction in IBS-SSS. Data, expressed as mean \pm standard error, were compared statistically before and after treatment using paired t-tests and non-parametric tests where applicable.

Results 44 South Asian patients with IBS (age $49 \pm 1.9$ years; 29 (66\%) female; 22/44 IBS-D, 16/44 IBS-C and 7/44 IBS-M; 15/44 Indian origin, 25/44 Pakistani origin, 4/44 other Asian countries; baseline IBS-SSS $332.8 \pm 14.3$ ) completed GFH. 40/44 (91\%) received GFH in English. Overall, 37/44 (84\%) achieved a $\geq 50$-point reduction in IBS-SSS and 25/44 (57\%) achieved $\geq 30 \%$ reduction in abdominal pain scores. Following 12-sessions of GFH, there were significant mean improvements in IBS-SSS $(-132.1, \mathrm{P}<0.0001)$, non-colonic symptom score $(\mathrm{P}<0.0001)$, QoL score $(\mathrm{P}<0.0001)$, HAD-anxiety $(\mathrm{P}<0.0001)$ and HAD-depression $(\mathrm{P}<0.0001)$, compared to baseline. Response rates were similar in patients of Indian 12/ 15 (80\%) and Pakistani 23/25 (92\%) origin, $\mathrm{P}=0.32$. Although not statistically significant, there was a trend towards a higher response rate in patients treated by a South Asian therapist, compared to those treated by a non-Asian therapist (response rates: $21 / 23(91 \%)$ vs. $16 / 21$ (76\%), $\mathrm{P}=0.21)$.

Conclusion These data suggest that GFH is highly-effective in this population and support its development for IBS in South Asian countries. Regardless of the ethnicity of the therapist, response rates were similar to published GFH outcomes in other IBS populations. However, knowledge of the patients cultural beliefs and language may help customise the treatment, enhance the patient experience, and further optimise outcomes. 